\title{
Interacting Multiple Cracks
}

\author{
X.L. Han and T.C. Wang \\ LNM, Institute of Mechanics, Chinese Academy of Sciences, Beijing 100080, China
}

Keywords: Multiple Cracks, Damage

\begin{abstract}
A method is presented to solve the plane elastic problem of a solid weakened by numerous cracks. The method provides an efficient computational procedure to solve crack interaction problems. Besides non-intersecting multiple open cracks, the method can treat intersectional cracks, surface contact cracks or even partial surface contact cracks. All of these complicated multiple crack problems can be solved accurately.
\end{abstract}

\section{INTRODUCTION}

The need for solutions to damage problems of brittle materials has stimulated the interest of many researchers in studying the fracture of solids containing multiple cracks. In the micromechanical field, besides various indirecting effective medium methods, the direct analysis to the problem of multiple interacting cracks has become an area of research interest in recent years. Several methods have been proposed [1-4].

This paper presents a general method to solve the plane elastic problem of a solid weakened by a set of straight arbitrarily distributed cracks or some kinds of periodically distributed cracks. Using this method, cracks are presented by continuously distributed dislocations, and the complex stress functions of cracks are expressed as a series. By a superposition technique and a crack surfaces collocation method, the multiple crack problem is reduced to algebraic equations about coefficients of the series.

\section{BASIC FORMULAE}

It is well known that stresses and displacements for a homogeneous elastic body under plane deformation can be represented by two complex potentials. For convenience, potentials $\Phi(z)$ and $\Omega(z)$ will be used. Stresses can be derived from

$$
\begin{gathered}
\sigma_{x}+\sigma_{y}=2[\Phi(z)+\overline{\Phi(z)}] \\
\sigma_{y}-\mathrm{i} \sigma_{x y}==\Phi(z)+\Omega(\bar{z})+(z-\bar{z}) \overline{\Phi^{\prime}(z)}
\end{gathered}
$$

A crack can be considered as a continuous distribution of infinitesimal edge dislocation. For a single crack between $x=-a$ and $x=a$ on the $\mathrm{x}$-axis, the complex potentials are given by the following formula:

$$
\Phi(z)=\Omega(z)=\frac{\mu}{\pi \mathrm{i}(\kappa+1)} \int_{-a}^{a} \frac{\mathrm{b}(s) \mathrm{d} s}{z-s}
$$


where $\mathbf{b}(s)=b_{\mathrm{T}}(s)+\mathrm{i} b_{\mathrm{II}}(s)$ is the complex dislocation density at point $z=s$ on the crack surface.

Now express the dislocation density as a Chebyshev polynomial series:

$$
\mathrm{b}(s)=\frac{\mathrm{i}(\kappa+1)}{\mu} \sum_{0}^{\infty} \alpha_{m}\left(1-\tau^{2}\right)^{-\frac{1}{2}} T_{m}(s / a)
$$

where $T_{m}\left(\right.$ ) are Chebyshev polynomials of the first kind, $\alpha_{m}$ are complex unknowns to be determined.

Then the complex potentials for the crack in the local system can be expressed as:

$$
\begin{aligned}
\Phi(z)=\Omega(z) & =\frac{\mu}{\pi \mathrm{i}(\kappa+1)} \int_{-a}^{a} \frac{\mathbf{b}(s) \mathrm{d} s}{z-s} \\
& =\sum_{m=0}^{\infty} \alpha_{m}\left(\frac{z}{a}-\sqrt{\frac{z^{2}}{a^{2}}-1}\right)^{m} / \sqrt{\frac{z^{2}}{a^{2}}-1}
\end{aligned}
$$

Substituting Eq.(4) into Eq.(1), the stress field at any point due to the crack can be expressed as a series. Especially the stress field on the crack surface can be expressed as

$$
\sigma_{y}(x)-\mathrm{i} \sigma_{x y}(x)=\Phi^{+}(x)+\Omega^{-}(x)=-2 \sum_{0}^{\infty} \alpha_{m} U_{m-1}(x / a)
$$

where $U_{m}($ ) are Chebyshev polynomials of the second kind.

\section{A SET OF ARBITRARY CRACKS}

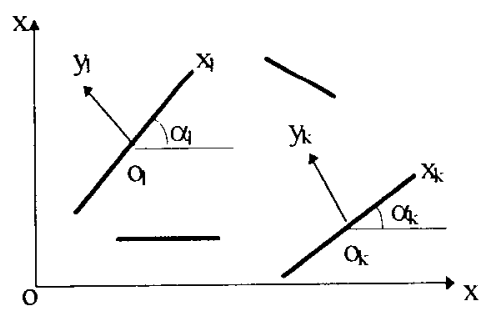

Fig 1 A set of artitrary cracks

An infinite linear elastic plane containing $N$ cracks is subject to remote uniform loading. Besides a global Cartesian coordinate system $O x y$, a set of local Cartesian systems $O_{k} x_{k} y_{k}(k=1,2, \cdots, N)$ situated at the center of each crack is employed with $x_{k}$ axis coincident with the crack surface. The $k$-th crack center situates at $C_{k}$, the crack length is $2 a_{k}$ and the crack surface makes angle $\alpha_{k}$ with the $O x$-axis, see Fig. 1 .

The problem can be represented as a superposition of $N$ individual crack problems, each involving only one isolated crack but loaded by unknown tractions, and with the superposition of all tractions on the crack surface being free (supposing the crack surface being open).

The traction along the 1 -th crack surface produced by remote loading is

$$
\sigma_{y l}^{(0)}-\mathrm{i} \sigma_{x y l}^{(0)}=2 \Gamma+\overline{\Gamma^{\prime}} e^{-2 \mathrm{j} \alpha_{l}}, \quad l=1,2, \cdots, N .
$$

where $\Gamma=\left(\sigma_{x}^{\infty}+\sigma_{y}^{\infty}\right) / 4, \quad \Gamma^{\prime}=\left(\sigma_{y}^{\infty}-\sigma_{x}^{\infty}\right) / 2+\mathrm{i} \sigma_{x y}^{\infty}$.

The stresses produced by the k-th crack in the local coordinate system $O_{k} x_{k} y_{k}$, from the last section, take the forms

$$
\begin{gathered}
\sigma_{x k}+\sigma_{y k}=2\left[\Phi_{k}\left(z_{k}\right)+\overline{\Phi_{k}\left(z_{k}\right)}\right] \\
\sigma_{y k}-\mathrm{i} \sigma_{x y k}=\Phi_{k}\left(z_{k}\right)+\Omega_{k}\left(\overline{z_{k}}\right)+\left(z_{k}-\overline{z_{k}}\right) \overline{\Phi_{k}^{\prime}\left(z_{k}\right)}
\end{gathered}
$$


where

$$
\Phi_{k}\left(z_{k}\right)=\Omega_{k}\left(z_{k}\right)=\sum_{m=0}^{\infty} \alpha_{k m}\left(\frac{z_{k}}{a_{k}}-\sqrt{\frac{z_{k}^{2}}{a_{k}^{2}}-1}\right)^{m} / \sqrt{\frac{z_{k}^{2}}{a_{k}^{2}}-1}
$$

And on the crack surface

$$
\sigma_{y k}\left(x_{k}\right)-\mathrm{i} \sigma_{x y k}\left(x_{k}\right)=\Phi^{+}\left(x_{k}\right)+\Omega^{-}\left(x_{k}\right)=-2 \sum_{m=0}^{\infty} \alpha_{k m} U_{m-1}\left(x_{k} / a_{k}\right)
$$

According to the formula of the coordinate system transformation, the tractions along the l-th crack surface in local coordinate system $O_{l} x_{l} y_{l}$ produced by the k-th crack can be written as follows

$$
\sigma_{y l}^{(k)}\left(z_{l}\right)-\mathrm{i} \sigma_{x y l}^{(k)}\left(z_{l}\right)=\frac{1}{2}\left[\sigma_{x k}\left(z_{k}\right)+\sigma_{y k}\left(z_{k}\right)\right]\left(1-e^{-2 i \alpha}\right)+\left[\sigma_{y k}\left(z_{k}\right)-i \sigma_{x y k}\left(z_{k}\right)\right] e^{-2 i \alpha}
$$

where $\alpha=\alpha_{l}-\alpha_{k}, \quad z_{k}=\left(C_{l}-C_{k}\right) e^{-\mathrm{i} \alpha_{k}}+z_{l} e^{\mathrm{i}\left(\alpha_{l}-\alpha_{k}\right)}$.

Along each individual crack surface, the tractions include that induced by the other cracks, the remote loading and the dislocations on the crack line. The traction-free condition on each crack surface can be written as follows

$$
\sum_{k=0}^{N}\left[\sigma_{y l}^{(k)}\left(x_{l}\right)-i \sigma_{x y l}^{(k)}\left(x_{l}\right)\right]=0,\left|x_{l}\right|<a_{l}, l=1,2, \cdots, N
$$

This is the governing equation for multiple crack problems.

\section{CALCULATION PROCEDURE}

Usually it is difficult to solve the governing equations (11) analytically. We use the boundary collocation method on crack surfaces, and truncate the potential series (8) to $M_{k}$ terms. The governing equations (11) are reduced to a system of linear algebraic equations for the unknown coefficients $\alpha_{k m}$.

Additionally, from the displacement single-valuedness condition, we have

$$
\alpha_{k 0}=0, \quad k=1,2, \cdots, N
$$

When the algebraic equations are solved, the complex potentials and the stress components produced by each crack are known. By superposition, the stress fields produced by the multiple cracks can be obtained.

\section{PERIODICALLY DISTRIBUTED CRACKS}

For an infinite solid containing some kind of periodic cracks, such as an infinite row of parallel equal cracks, doubly periodic equal cracks, or even the case of a collection of identical cells which contains some arbitrary cracks, the problem can be solved in a similar manner.

For the case of a row of parallel cracks or doubly periodic cracks, each individual crack has an identical situation. In the local system of each crack, the complex potentials is the same, so it can be expressed in the same way:

$$
\Phi_{k}\left(z_{k}\right)=\Omega_{k}\left(z_{k}\right)=\sum_{m=0}^{\infty} \alpha_{m}\left(\frac{z_{k}}{a}-\sqrt{\frac{z_{k}^{2}}{a^{2}}-1}\right)^{m} / \sqrt{\frac{z_{k}^{2}}{a^{2}}-1}
$$

where $z_{k}=\left(z-C_{k}\right) e^{-i \alpha_{k}}$.

Since each crack has an identical situation in its local system, we can take any one crack into consideration. 


\section{INTERSECTED CRACKS}

Though this paper proceeds on non-intersecting cracks, with appropriate corrections, this method can also be used to treat intersecting cracks. For example, a bent crack can be treated as two straight intersecting cracks. Instead of the displacement single-valuedness condition for each crack, we use the condition for the bent crack, together with the condition of the stress intensity factor vanishing at the intersecting point.

\section{CLOSED AND PARTIALLY CLOSED CRACKS}

In a multiple crack system, for strong crack interactions, crack surfaces are not always open even under remote tension loading. Some crack surfaces may be in contact or partially contact.

For a contact sliding crack (without friction), the crack is represented as a distribution of Type II dislocations. The dislocation density function $b_{\text {II }}$ is expressed as a series similar to that of the open crack, but with real coefficients.

For a partial contact crack, it is represented as a distribution of complex dislocations with both Type I and Type II dislocations. The two types of dislocations are considered separately. The Type II dislocation density $b_{\mathrm{II}}$ and the complex potentials reduced by it are treated in the same way as a contact crack. Assuming that the open part of the crack is $L \in(c, a),-a<c<a$, express the Type I dislocation density $b_{\mathrm{I}}$ on this part as:

$$
b_{\mathrm{T}}(s)=\frac{\mathrm{i}(\kappa+1)}{\mu} \sum_{0}^{\infty} \beta_{m}\left(1-\tau^{2}\right)^{-\frac{1}{2}} T_{m}(\tau)
$$

where $s=\frac{1}{2}[(a-c) \tau+a+c], \beta_{m}$ are real series coefficients to be determined.

The complex potentials for this Type I dislocations distribution are expressed as:

$$
\begin{aligned}
\Phi(z)=\Omega(z) & =\frac{\mu}{\pi \mathrm{i}(\kappa+1)} \int_{c}^{a} \frac{b_{\mathrm{I}}(s) \mathrm{d} s}{z-s} \\
& =\sum_{m=0}^{\infty} \beta_{m}\left(\zeta-\sqrt{\zeta^{2}-1}\right)^{m} / \sqrt{\zeta^{2}-1}
\end{aligned}
$$

where $\zeta=(2 z-a-c) /(a-c)$.

For a partial contact crack, the contact length should be determined. The normal traction should vanish at the point from open to contact zone. It seems reasonable to eliminate the Type I stress intensity factor at that point. This provides an auxiliary condition for the determination of contact length.

The calculation procedure is similar, but the crack surface conditions are different for open and non-open cracks: for open cracks, both normal and shear tractions are free; for contact sliding cracks (without friction), shear tractions are free; for partial contact cracks (without friction), shear tractions are free, normal tractions are free only on open parts.

For a solid with multiple cracks under remote loadings, perhaps some crack surfaces are open, some others are in contact or partially in contact. Since crack conditions are not known in advance, all crack surfaces are considered to be open first, and the multiple cracks problem is obtained using the traction free conditions for open cracks as before. Then according to the calculated stress intensity factors at crack tips and displacement conditions on crack surfaces, the conditions of crack states are checked. If they are reasonable, the problem is solved. Otherwise, the assumption of crack conditions is changed until reasonable result is obtained. 


\section{EXAMPLES, RESULTS AND DISCUSSION}

For two equal collinear cracks in an infinite plate under remote tension or shear loading. The normalized stress intensity factors with an accuracy of six digits, and the terms $M$ of truncated complex potentials series (to assure accuracy) for different crack spaces, are shown in table 1.

Table 1 SIF $K=k / \sigma \sqrt{\pi a}$ for different $2 a / b$ (crack length/crack center space).

\begin{tabular}{|cccc|cccc|}
\hline $2 a / b$ & $M$ & $K$ (out tip) & $K$ (inner tip) & $2 a / b$ & $M$ & $K$ (out tip) & $K$ (inner tip) \\
\hline 0.10 & $(3)$ & 1.00120 & 1.00132 & 0.60 & $(7)$ & 1.04094 & 1.08040 \\
0.20 & $(4)$ & 1.00462 & 1.00566 & 0.70 & $(9)$ & 1.05786 & 1.13326 \\
0.30 & $(4)$ & 1.01017 & 1.01383 & 0.80 & $(14)$ & 1.08107 & 1.22894 \\
0.40 & $(5)$ & 1.01787 & 1.02717 & 0.90 & $(18)$ & 1.11741 & 1.45387 \\
0.50 & $(6)$ & 1.02795 & 1.04796 & 0.99 & $(55)$ & 1.20553 & 2.99397 \\
\hline
\end{tabular}

To show the validity of this method in treating multiple crack problems, we give an example of a plane which contains as many as a hundred cracks. The calculations are carried out for an array of equal collinear cracks of length $2 a$ with equal spacings of $b$. The crack numbers $N$ can be up to a hundred. The maximum stress intensity factors are shown to occur at the middle crack tips and given in Table 2 for the case of $2 a / b=0.5$. The case $N \rightarrow \infty$, that is periodic distributed cracks, is also calculated.

Table $2 K=k / \sigma \sqrt{\pi a}$ for different numbers of collinear cracks with $2 a / b=0.5$.

\begin{tabular}{cccccc}
\hline$N$ & 21 & 31 & 41 & 51 & 61 \\
\hline$K$ & 1.120772 & 1.123233 & 1.124493 & 1.125257 & 1.125771 \\
\hline$N$ & 71 & 81 & 91 & 101 & $\infty$ \\
\hline$K$ & 1.126139 & 1.126416 & 1.126632 & 1.126806 & 1.128379 \\
\hline
\end{tabular}

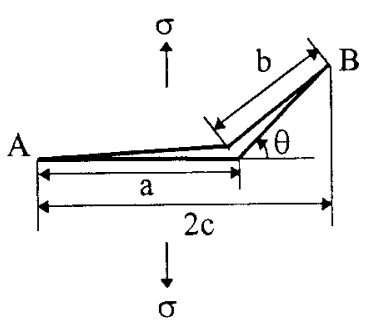

Fig. $2 \mathrm{~A}$ bent crack under tension

For intersecting cracks, a bent crack example is given. The bent crack with branch angle $\theta=45^{\circ}$ and varying branched crack lengths, is under tension loading, see Fig 2.

The calculated normalized stress intensity factors are compared with those shown in the Handbook of Stress Intensity factors [5], and tabulated in Table 3 . It is shown that they agree well.

Table 3 Stress intensity factors $K_{i}=k_{i} / \sigma \sqrt{\pi c}$ for a bent crack with branch angle $\theta=45^{\circ}$.

\begin{tabular}{|c|c|c|c|c|c|c|c|c|}
\hline & \multicolumn{2}{|c|}{$K_{1}^{A}$} & \multicolumn{2}{c|}{$-K_{2}^{A}$} & \multicolumn{2}{c|}{$K_{1}^{B}$} & \multicolumn{2}{c|}{$K_{2}^{B}$} \\
\hline $2 a / b$ & Present & Isida & Present & Isida & Present & Isida & Present & Isida \\
\hline 0.10 & 0.9982 & 0.998 & 0.0186 & 0.018 & 0.6329 & 0.634 & 0.5042 & 0.505 \\
\hline 0.20 & 0.9951 & 0.995 & 0.0280 & 0.028 & 0.5980 & 0.598 & 0.5575 & 0.558 \\
\hline 0.50 & 0.9876 & 0.988 & 0.0326 & 0.032 & 0.5697 & 0.570 & 0.6197 & 0.619 \\
\hline 0.80 & 0.9848 & 0.985 & 0.0238 & 0.024 & 0.5677 & 0.568 & 0.6374 & 0.637 \\
\hline 1.00 & 0.9848 & 0.985 & 0.0158 & 0.016 & 0.5693 & 0.569 & 0.6413 & 0.641 \\
\hline
\end{tabular}




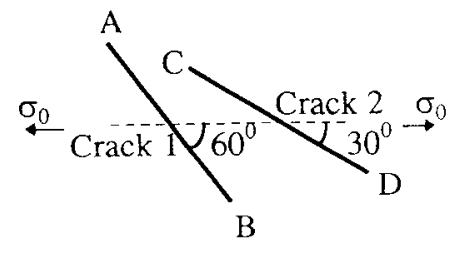

Fig. 3 The configuration of two cracks

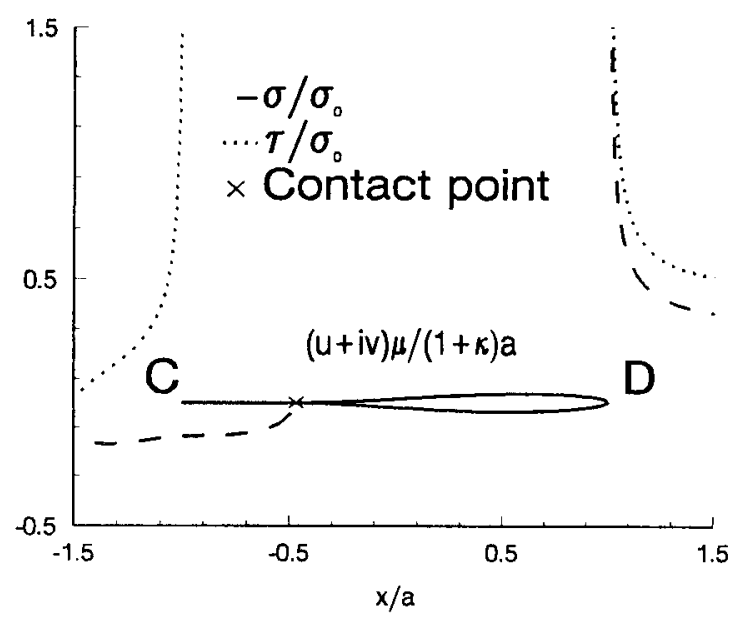

Fig. 4 Crack 2 displacement and stresse conditions

An example of a partially closed crack is given now. A solid with two equal cracks (half length $a$ and crack center space $a$ ) under remote tension is shown in Fig. 3. First, assuming the surfaces of the two cracks are open, the calculated stress intensity factors show that the Type I stress intensity factor at one tip of Crack 2 is negative. Then, assuming Crack 2 is a partial contact one. The calculated crack surface displacements and stresses on crack lines (see Fig. 4 for crack 2) are shown to be correct now.

Since the present research provides a unified and efficient method to solve multiple interacting crack problems, it may be used as an efficient tool to study the damage procedure of brittle materials, from cracks initiation to propagation till last failure of materials. Certainly, there are lots of problems in need of further study.

\section{References}

1. M.Kachanov, Int. J. Fracture, 28 (1985), p.R11.

2. H.Hori and S.Nemat-Nasser, Int. J. Solids Structure, 21 (1985), p.731.

3. Y. Benveniste, G.J. Dvorak, J. Zarzour and E.C.J. Wung, Int. J. Solids Structure, 25 (1989), p.1275.

4. X.L. Han and T.C. Wang, Progress in Advanced Materials and Mechanics, eds. T.C. Wang and T.W. Chou, Peking University Press, Beijing, 1996, p.688.

5. Y.Murakami, Stress intensity factors handbook, Pergamon press, World Pub. Corp., Beijing, 1989, p.352. 
Fracture and Strength of Solids III

10.4028/www.scientific.net/KEM.145-149

\section{Interacting Multiple Cracks}

10.4028/www.scientific.net/KEM.145-149.381 